\title{
DECISIONS THEORY IN EARTHQUAKE ENGINEERING
}

\author{
E. Rosenblueth*
}

\section{Introduction}

Let us choose to be rational. As designers we shall aim to produce the best designs possible - to optimize。

To optimize from whose point of view? -ours, of course. This does not mean we should ignore society, the tenants, or the owner, nor even the architect and the contractor; their interests mouldour own. For the sake of simplicity I shall assume that our values coincide with those of society. If the owner's, the architect's, or the contractor's do not, why too bad for them, or for us. The present treatment should serve as a guide to take the compromise decisions that real situations force upon us; and it is directly applicable when we are employed by a branch of a government that represents the people.

We wish then to maximize utility for society. This is equivalent to maximizing an objective function equal to the expected present value of the benefits derived from the existence of the structure minus all the expenditures, including initial cost, maintenance, and the consequences of damage and failure. These quantities should be adjusted for nonlinear relations between money and utility whenever there is an appreciable nonlinearity.

For the sake of simplicity we shall assume that structures are built instantly and that. when they suffer damage or collapse, they are also repaired or rebuilt instantly. Let us begin counting time when the structure is born. At a future instant $t$ an earthquake may occur or there may be a change in live load. The effects of these phenomena depend on the state in which they find the structure at time $t$. That state is a function of the structure's initial properties, of the damage wrought by earthquake, gravity, and other loads, and of the repairs done in the interval (o.t). This wording suggests that the sequence of damagerepairs-failures may be regarded as a Markov chain and indeed such an approach is fruitful (1). Nevertheless, we shall use a more straight-forward type of analysis.

In the present simplified treatment we shall consider the superposition of two independent stochastic processes: gravity loads and the effects of earthquakes: we sha11 recognize that seismic forces depend on live load. Our study will be limited to structures having a single degree of freedom. We shall leave for some other occasion our concern with more realistic idealizations of the disturbances,

* Professor of Engineering, Universidad Nacional Autónoma de México, México, D F, Mexico. with wind as a third stochastic process, with the natural evolution of structural properties, and with the extension of the theory to structures having several degrees of freedom.

\section{Reliability Function and Discount Function}

Before dealing explicitly with optimization we deem it convenient to present some basic relations taken from reliability theory 2) and discuss the matter of present values.

The properties of a system are described by the vector of its parameters, $\{x\}$. Every term of this vector is a parameter of the probability distribution of one of the structure's properties. Those $x_{i}$ "s which come under the designer's control are called design parameters. Thus, if one of the structural components is a bar that will be subjected to tension, one of the $x_{i}$ 's may be a central value (expectation, median, mode, etc) of the probability distribution of the bar's strength in tension while another $x_{i}$ may be a second parameter (such as standard deviation or coefficient of variations) of that distribution. These will be all the parameters associated with the strength of that bar if its distribution can be completely described by two parameters; otherwise there will be other xi "s associated with that strength. Often the central value will be selected by the designer and so it will be a design parameter. Alternatively the design parameter may be another functional of the distribution, such as a "characteristic," nominal or "minimum guaranteed" value, or they may be two design parameters.

Consider a system that fails either upon completion or not at all. Let $F$ denote its probability of failure. It is a function of $\{x\}$. Then $R(\{x\})=1-F(\{x\})$, its probability of survival, is known as the system's reliability.

If the system can fail at times other than the initial one, we shall let $F(\{x\}, t)$ denote the probability that it fail in the time interval $(0, t)$. $F(\{x\}, t)$ is consequently the probability distribution function of the time to failure and is known as the failure distribution function. $\left.R(\{x\}, t)=1 \frac{1}{F(\{x\}}, t\right)$ is the probability that the system has survived up to time $t$; it is known as the reliability

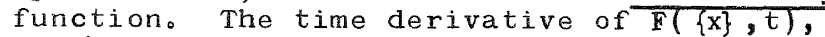
$f(\{x\}, t)$, is the failure density function, or probability density function of the time to failure.

The unconditional probability that failure occur in the interval $(t, t+d t)$ is $f(\{x\}, t) d t$. The conditional probability that it occur in this interval given that it has not taken place 
at an earlier time is $\nu(\{x\}, t) d t$, where

$$
\nu(\{x\}, t)=\frac{f(\{x\}, t)}{R(\{x\}, t)}
$$

is the hazard function or risk function. can be shown from eq 1 that

$$
R(\{x\}, t)=\exp \left[-\int_{0}^{t} \nu(\{x\}, \gamma) d \tau\right]
$$

The hazard function can also be interpreted as the expected rate of failure occurrence, and its reciprocal as the return period of failures.

In particular, a constant failure rate, $\nu(\{x\}, t)=\nu(\{x\})$, defines a Poisson process for a given vector $\{x\}$. It defines a generalized Poisson process when we consider a family of $v e c t o r s\{x\}$. According to eq 2 the process is associated with the reliability function

$$
R(\{x\}, t)=e^{-\nu(\{x\}, t) t}
$$

and hence with a failure distribution function 1 - $e^{-\nu t}$ and with a failure density function $\nu e^{-\nu t}$.

On the matter of present values, it is obvious that normally we assign a higher value to short-range than to long-range benefits and losses. We therefore introduce a monotonically decreasing function of time, $\Delta(t)$, which we shall call the discount function. This function, when multiplied by a future benefit or loss, gives its present value, that is, the value it would have for us if we received or incurred in it at present. The discount function reflects the availability of resources, uncertainty about the future, and concern for the familiar, and it depends on the subject for whom we wish to compute utilities and optimize decisions。

If the subject's values coincide with those of society it is licit to assume that $\Delta(t)$ is stationary over a fairly large time span. It follows that without undue error we may take

$$
\Delta(t)=e^{-\delta t}
$$

where $\delta$ is a constant rate of continuous interest and we begin counting time at present. This rate is related with that of yearly interest, say $6 \cdot$ at which financial matters are operated, by the equation $\delta=1 n(1+\delta$ ') since a yearly interest would lead to a discount function

$$
\Delta^{\prime}(t)=\left(1+\delta^{\prime}\right)^{t}
$$

When $\delta^{\prime} \ll 1$ we find $\delta \cong \delta^{\circ}$.

The discount function can also be interpreted as follows. If we deposit an amount $B_{Q}=\Delta(t) B$ at time zero at a continuous rate of compound interest $\delta$, after a time $t$ we will have an amount $B$ deposited.

\section{Constant Gravity Loads; Single Failure Mode}

We shall begin by considering a very simple model. The structure we have in mind fails when the numerical value of one of its structural responses, $Y$ (say, its displacement relative to the ground or its absolute acceleration), exceeds a limit, $x$, which we shall refer to as the structure's resistance. We shall take $X$ to be a time independent random variable. We shall assume that there is a single design parameter, $x$, which is a functional of the probability distribution of $\mathrm{X}$ 。

Given the computed value, $z$, of some parameter of a ground motion, we can compute the probability distribution of its actual value, $\mathrm{Z}$ (see ref 3, for example)。 (A pertinent such parameter may be the maximum ground acceleration, velocity, or displacement or the mean value of some spectral ordinate over a range of natural periods of vibration.)

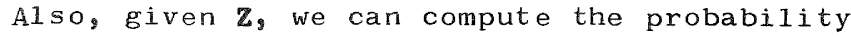
distribution of the structural response, $Y$ (see refs 4 and 5, for example). Finally, for a given value of $z$ we can obtain, in a theoret ical model of seismicity, the rate $\lambda(z)$ with which this parameter is exceeded $(3,5,6)$. We idealize the series of such temblors as a Poisson process, so that, regarding $z$ as a variable, earthquakes felt at a station constitute a generalized Poisson process. It follows that $\lambda(z)$ is independent of $t$.

Using the identity

$$
z \equiv(z / Z)(Z / Y)(Y / X)(X / x) x
$$

we can write

$$
\begin{aligned}
\mathrm{P}(\text { failure }) & =\mathrm{P}\left(\frac{\mathrm{Y}}{\mathrm{X}}>1\right) \\
& =\mathrm{P}(\mathrm{z}>\phi \mathrm{x})
\end{aligned}
$$

where $P(\cdot)$ denotes the probability of occurr ence of the event in parenthesis and $\phi$ is given by

$$
\phi=\phi_{x} \phi_{y} \phi_{z}
$$

with

$$
\begin{aligned}
& \phi_{x}=x / x \\
& \phi_{y}=z / y \\
& \phi_{z}=z / z
\end{aligned}
$$

If we knew the value of $\phi$ we could put the rate of structural failures in the form

$$
\begin{aligned}
\nu(x) & =\lambda(z) \\
& =\lambda(\phi x)
\end{aligned}
$$

Actually $\phi$ is a random variable and hence so is $\nu(x)$. Usually without excessive error the random variables $\phi_{x, y}$ can be taken as stochastically independent. Their probability distributions are reasonably well behaved. The distribution of $\phi_{x}$ is approximately lognormal, since $X$ can be expressed as the product of several nearly independent variables having well behaved distributions. That of $\phi_{\mathrm{z}}$, which ordinarily involves the greatest dispersion, is roughly also lognormal (3). In most cases, then, we can assign in $\phi$ a normal distribution with expectation and variance equal to the sums of the expectations and variances of the logarithms of $\phi_{x}, \phi_{y}$, and 
$\phi_{z}: \phi=\exp [E(\ln \phi)]$ is the mode of $\phi$. In the same fashion we can find the parameters of the distribution of $1 \mathrm{n} \phi_{\mathrm{x}}$ while those of the distribution of $1 \mathrm{n} \phi_{\mathrm{y}}$ can be computed from known approximations to the distribulion of $\phi_{y}^{-1}(4,5)$. For greater accuracy or generality we can always compute the probability distribution of $\phi$ using numerical integration or a simulation procedure based on the combination of random numbers having the distribulions of the independent variables. The latter approach is similar to the one which has been used in calculating the probability distributions of resistance functions of timber ( 7$)$ and reinforced concrete (8) beams. Consequentiy we can always evaluate eq 9 with the degree of accuracy that we may desire.

Once we have computed the rate of structural failures we can proceed to obtain the expected present value of losses due to failure, which we shall need to optimize design. Let $D$ be this value and $H$ denote the loss incurred in case of failure. Then,

$$
\begin{aligned}
& D=E\left(\int_{0}^{\infty} F f \Delta d t\right) \\
& =E\left[\nu \int_{0}^{\infty} H e^{-(\nu+\delta) t} d t\right]
\end{aligned}
$$

When the expectation of $H$ can be taken as time independent, eq 10 becomes

$$
D=H E\left(\frac{v}{v+\delta}\right)
$$

This expression is valid if the structure is to be abandoned after failure. If, however, it is to be systematically rebuilt we find

$$
\begin{aligned}
D & =(C+H) \sum_{i=1}^{\infty}\left[E\left(\frac{\nu}{\nu+\delta}\right)\right]^{i} \\
& =(C+H) \Psi
\end{aligned}
$$

where

$$
\Psi=\frac{E\left[\frac{\lambda(\phi x)}{\lambda(\phi x)+\delta}\right]}{1-E\left[\frac{\lambda(\phi x)}{\lambda(\phi x)+\delta}\right]}
$$

and $\mathrm{C}$ is the structure's expected initial cost (9). The expectation is to be computed with $\phi$ as the random variable.

If the structure is to be abandoned after failure, benefits derived from its existence cease to be produced at the moment of failure. Hence, the loss is greater than indicated by eq 11. If it is to be rebuilt systematically, the interruption in the production of benefits is temporary and should be included in $\mathrm{H}$ in eq 12. while any salvage value should be deduced therefrom. If, as assumed in this expression, the cost of rebuilding is the same as the initial capital investment, we find that every structure that was worth building in the first place is always worth rebuilding after failure (this is a consequence of our assumption of stationarity in the failure process).

In some practical cases the cost of rebuilding far exceeds the cost of originally building the structure. The situation may arise in dams and other struclures when failure involves large landslides or when it seriously affects the foundation.

In what follows we shall confine our attention to cases in which eq 12 is applicable.

We shall take $C+D$ as our objective function, since in most cases the benefits derived from the structure's existence are nearly insensitive to $x$. Optimization amounts then to the choice of that value of $x$ which minimizes $C+D$.

In the range of interest it is often sufficiently accurate to take $C$ of the form

$$
c=a+b x
$$

in which $\underline{a}$ and $b$ are constants. Assume further that $\lambda$ is of the form

$$
\lambda=\mathrm{Bz}^{-\gamma}
$$

where $B$ and $\gamma$ are constants and $\gamma$ lies between 2 and 4 (3). Combining eqs 13 and 14 with the assumption that $\phi$ has lognormal distribution we can compute $C+D$ numerically as a function of $x$ and find the optimum $x$ by trial and error. Results are displayed in fig 1 for various values of the parameters involved.

As an example suppose that $z$ stands for maximum ground acceleration in terms of gravity, $r=2.5$, and $B=0.015$. Suppose al so that $X$ is the base shear coefficient which would make the structure fail and that it has lognormal distribution with mode $x$. Let $b / a=1.5, \mathrm{H} / \mathrm{a}$ $=10$, and $\delta=0.1 \mathrm{yr}^{-1}$. It is reasonable to assume $\phi=1.0$ and $\sigma=\sqrt{\operatorname{Var} \text { In } \phi}=0.3$. Then $x_{0}$, the optimum $x$, is 1.04 according to fig 1.

Application of these principles to structural systems has led to the development of reliability optimization in the design of statically determinate structures. The problem consists in assigning the optimum reliability to each member of the structure. It has been solved for the case in which the overall reliability is given and the structure is subjected to a single static application of a set of random loads (10) and for the case in which the overall reliability is not specified beforehand and the loads are random functions of time ( 9 ).

The same principles have led to a rational procedure for establishing bonuses and penalties to the price of concrete as a function of the material strength as determined from tests on control cylinders (11). This question is of interest primarily because the usual policy, when there is defective-strength concrete in a job under construction, is either to leave matters stand with perhaps some additional curing, or to strengthen or tear down and rebuild if understrength is very pronounced. In the first case the owner's utility is diminished because he gets a structure having a reduced reliability, while in the latter case there is a loss to the builder and to the owner because of the cost and delay of the operation, and in no case is the decision optimum for society. The policy of setting a rationally based penalty for under-strength and a (very small) bonus for overstrength is favourable to most of the parties concerned and usually acceptable to all. 
Approximate formulas for the calculation of reliability and for roliability optimization in various types of structural membors and structures under various kinds of loading have been worked out, chiefly with the aim of facilitating the adoption of an explicit reliability optimization format in building
codes $(12,13)$.

\section{Constant Gravity Loads: Damage and Failure States}

Consider now structures that may undergo a multistage process of failure, such as may result from the use of defense plateaus. The conscious formal adoption of this type of structural solution is due to Blume (14) The principle involved is that failure of certain structural elements, such as concrete-block or hollow-tile partitions, occurs at deformations smaller than those causing collapse of the entire structure, and retards it. There may be additional plateaus, such as those furnished by the concrete fire protection of steel rrames. Failure of each set of these relatively dispens. $a b l e$ structural elements spends part of the energy transmitted from the earthquake to the structure and herice there is a smaller probability of collapse. Whether the solution is advantageous depends on the expected frequency with which the structure will suffer damage and on the cost of this damage.

The shear-deformation curves for a structure of this type may take a shape similar to that in fig $2 a$ (see refs 15 and 16 for sheardeformation curves of confined masonry walls). The loss-deflection relations may resemble the full lines in fig $2 b$, which are partly based on rough cost estimates for loss in rentability or cost of repair, whichever is smaller, the estimates having been made from photographs and descriptions of walls tested in the laboratory. The curves may be idealized as a stepped function such as the dashed line in fig $2 \mathrm{~b}$.

We shall assume that immediately after every earthquake the structure is repaired returning it to its original state if it was damaged, and that it is instantaneously replaced with a new structure if it has collapsed. We assume that the resistance functions of the repaired and the new structures have the same probability distributions as those of the original structure and are independent therefrom. Schematically we may represent the possible states of the structure and the transitions between states as in fig. 3.

Let $C$ and $H$ denote, as before, the expected cost of building the structure and the expected loss in case of collapse; let $H_{i}$ denote the expected loss in case the structure enters the 1th state of damage, including the cost of repair, with $i=1,2, \ldots, n ;$ let $H_{n+1} \equiv H$; and let $x_{i}$ denote the nominal resistances associated with the various states of damage, and $x_{n+1}$ the nominal resistance associated with

* Treatment in ref 1 differs from the present formulation in that the former assumes that the repaired and the new structures are identical with the original. Thus, in ref 1 eq 12 becomes $D=(C+H) E(\nu / \delta)$ using our notation. The actual situation for a repaired structure is likely to 1 ie between both assumptions while the present formulation is clearly more appropriate for new structures. zollapse. Then the objective function we have chusen is

$$
\begin{aligned}
& C+D=C+\sum_{i=1}^{n} H_{i}\left[\Psi\left(x_{i}\right)-\Psi\left(x_{i+1}\right)\right]+ \\
& \left(C+H_{n+1}\right) \Psi\left(x_{n+1}\right)
\end{aligned}
$$

Ordinarily the optimum value of $x_{n+1}$ is considerably larger than the optimum $x$ one would rind without the defense plateaus. Hence, $\Psi\left(x_{n+1}\right)$ is ordinarily much smaller than the optimal $\Psi(x)$ and it is advantageous to adopt this solution provided the $\mathrm{H}_{j}$ 's and the differences $\Psi\left(x_{i}\right)-\Psi\left(x_{i+1}\right)$ are not too large.

When the optimal policy is not to repair after the structure has undergone minor damage, it suffices to write the expectation of $\lambda\left(\phi x_{i}\right) /\left[\lambda\left(\phi x_{i}\right)+\delta\right]$ instead of $\Psi\left(x_{i}\right)$ in eq 15 for the values of $i$ for which this is the case.

In place of the stepped function we have assumed in deriving eq 15, we can deal directly with a continuous curve relating $H$ and $x$, or with a curve having a finite number of discon. tinuities, provided we can assume that the relation between these variables is unique. We obtain

$$
\begin{aligned}
& C+D=C+\int_{0}^{x_{n+1}} H(x) \psi d x+ \\
& \left(C+H_{n+1}\right) \Psi\left(x_{n+1}\right)
\end{aligned}
$$

where $H(x)$ is the total damage undergone by the structure, including the cost of repair, when the nominal deformation is $x$, and $\psi=\psi(x)$ is $\mathrm{d} \Psi / \mathrm{dx}$. An obvious modification makes eq 16 applicable to structures for which the optimal policy consists in not repairing damages for smal1 values of $x$.

\section{A Deterministic Live-Load Model}

In order to bring out the main features of optimal design we shall adopt a particularly simple model of $11 \mathrm{ve}$ load. We shall assume that, given a value of $t$, there is a time independent probability, p, that the total static value of gravity loads be equal to $W_{f}$ and a probability 1 - p that it be equal to $W_{e}<W_{f}$. This is an idealization of the variation of gravity loads on auditoriums and similar structures when we disregard dynamic effects due to the motion of people.

The difference $W_{f}-W_{\theta}$ is due to people. Under the action of gravity alone, the total load at a given instant is either $W_{e}$ or $W_{\theta}+$ $\alpha\left(W_{f}-W_{\theta}\right)$ where $\alpha$ is an impact factor; is a random function of time. For the sake of simplicity we shall take $W_{\theta}$ and $W_{f}$ as deterministic and time independent and assume that $\alpha$ attains a deterministic maximum value shortly after completion of the structure.

We shall again call $\mathrm{C}$ the structure's expected initial cost. The loss in case of failure will now depend on the number of people present at the time of failure. We shall take this number to be either zero or deterministically equal to some value, proportional to $W_{f}$ - We. Accordingly, the loss due to fallure 
can be written as equal either to $\mathrm{H}_{e}$ or to $\mathrm{H}_{\mathrm{f}}$, where $H_{f}-H_{e}$ is proportional to $W_{f}-W_{e}$.

\section{Deteministic Variable Live Load Plus Earthquake}

Now we shall consider the combined action of variable gravity loads, conforming to the foregoing model, and earthquakes. Initially we shall confine our attention to structures that have a single failure state. We shall assume that the probabilily is negligible that $\alpha$ differ appreciably from 1 during the action of an earthquake having an appreciable probability of causing failure. (Actually wo can expect important dynamic effects due to live load during an earthquake but they are probably not very significant at instants of maximum seismic response. The matter deserves observations and study.) Hence, if $p$ denotes the probability that the auditorium is full at the time of such ground motion, the total load acting on the structure will be W with probability 1 - $p$, or with probability p. The loss in case of failure will be $H_{e}$ in the first case and $H_{f}$ in the second case. To simplify the notation we shall call $\propto$ the maximum value of $\alpha$.

For sufficiently small values of $t$ we can neglect the probability of occurrence of earthquakes having an appreciable probability of causing failure. If $\mathrm{X}_{\mathrm{g}}$ denotes the capacity of the structure to withstand gravity loads, the probability that the structure fail when vacant is

$$
P_{e}=P_{X_{g}}\left(W_{e}\right)
$$

and the probability that it fail with full auditorium is

$$
P_{f}=P_{X_{g}}\left[W_{e}+\alpha\left(w_{f}-w_{e}\right)\right]-P_{x_{g}}\left(w_{e}\right)
$$

(Notice that we are not committed to the assumption that either $W_{e}$ or $W_{f}$ are uniformly distributed over the loaded area. We do require, however, that the probability that the total gravity load exceeds the structural capacity, $X_{0}$, is uniquely defined by the magnitude of that load.)

Hence, the expected loss due to failure under gravity loads is

$$
D_{g}=H_{\theta} P_{\theta}+H_{f} P_{f}
$$

If the structure is rebuilt instantaneously, we must add $C\left(P_{e}+P_{f}\right) t_{Q}$ this expected loss and Multiply it by $1+\sum_{i=1}\left(P_{e}+P_{f}\right)^{i}$. Hence it becomes

$$
D_{g}=\frac{H_{e} P_{e}+H_{f} P_{f}+C\left(P_{e}+P_{f}\right)}{1-\left(P_{e}+P_{f}\right)}
$$

where $P_{e}+P_{f}=P_{X_{g}}\left[W_{e}+\alpha\left(W_{f}-W_{e}\right)\right]$.

We shall assume that the probability distribution of $x_{\text {g }}$ is defined by two parameters. We shall take one of these to be the nominal value of this variable and denote it by $x^{\circ}$ We assume that the designer has control on only this parameter of the distribution.

According to our assumptions the structure will be standing at the time of every earth- quake. The maximum basp shear is a function of the gravity loads, $W(5)$. For short natural periods (shorter than about $2 \pi \mathrm{v} / \mathrm{a}$ where a and $v$ are respectively the maximum numerical values of ground acceleration and velocity), the expected maximum base shear is approximately proportional to $W$. If the structure rests on firm ground, for intermediate natural periods (up to about $2 \pi \mathrm{d} / \mathrm{v}$ where $\mathrm{d}$ is the maximum numerical value of ground displacement) the expected maximum base shear varies approximate$1 \mathrm{y}$ as $\mathrm{w}^{1 / 2}$. And for very long natural periods (longer than about $2 \pi \mathrm{d} / \mathrm{v}$ if the structure rests on firm ground) it is practically independent of $W$. What we have said of shear applies as well to other responses。

The probability distribution of a response normalized with respect to its expectation depends little on the gravity load when the structure stands on firm ground (5). The probability of failure during a given earthquake, under constant gravity load, is $P(Y>X)$ where $Y$ is the response and $X$ the capacity to resist earthquakes. Therefore, if $\beta=$.... $E(Y) / E\left(Y_{O}\right)$ is a nondecreasing function of $W$, $Y_{0}$ denotes the response of a reference structure having a gravity load, say $W_{0}$, and the capacity of the actual structure to resist earthquakes is $X-G_{\text {g }}$ where $G$ is a nonincreasing function of load, then the probability of failure is $P\left(Y_{O}>U\right)$, where

$$
U=\frac{X-G(W)}{\beta(W)}
$$

We wish to incorporate the information derived from the fact that, prior to the earthquake, the structure had withstood a gravity load, say equal to $W_{1}$, where $W_{1}=W_{\theta}+$ $\alpha\left(W_{f}-W_{e}\right)$. This fact guarantees that $X$ is not smaller than $G\left(W_{1}\right)$. Hence, if $\mathrm{P}_{\mathrm{X} / \mathrm{W}_{1}}(\cdot)$ denotes the probability distribution function of $\mathrm{X}$ given that the structure has withstood the load $W_{1}$, we have

$$
P_{X \mid W_{1}}(X)=\left\{\begin{array}{l}
0 \text { if } X \leq G\left(W_{1}\right) \\
\frac{P_{X}(X)-P_{X}\left[G\left(W_{1}\right)\right]}{1-P_{X}\left[G\left(W_{1}\right)\right]} \text { if } X>G\left(W_{1}\right)
\end{array}\right.
$$

Now let $u(w)$ denote the value of $U(w)$ when we replace $X$ with $x$ in $E q 20$. Then the rate of failure is

$$
(1-p) \nu\left[u\left(w_{e}\right)\right]+p \nu\left[u\left(w_{f}\right)\right]=\lambda_{\theta}+\lambda_{f}
$$

where

$$
\begin{gathered}
\lambda_{e}=(1-p) \lambda\left[\phi_{e} u\left(w_{e}\right)\right], \lambda_{f}=p \lambda\left[\phi_{f}\left(w_{f}\right)\right] \\
\phi_{e}=\phi_{u e} \phi_{y} \phi_{z}, \phi_{f}=\phi_{u f} \phi_{y} \phi_{z} \\
\phi_{u \theta}=\frac{u\left(w_{\theta}\right)}{u\left(w_{\theta}\right)}
\end{gathered}
$$

and

$$
\phi_{u f}=\frac{u\left(w_{f}\right)}{u\left(w_{f}\right)}
$$

It follows that the expected present value of the loss due to failure under earthquake is 


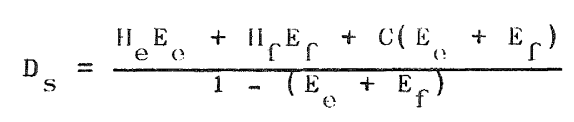

where $E_{e}$ and $E_{f}$ are $\left.E[\lambda / \alpha+\delta)\right]$ for $\lambda$ equal to $\lambda_{e}$ and $\lambda_{f}$ respectively.

Now we can calculate the objective function by combining eqs 19 and 21 for the lotal expected loss due to railure:

$$
C+D=C+D_{g}+D_{S}
$$

Finally, if we express $\mathrm{C}$ in terms of $\mathrm{x}_{\mathrm{g}}$ and
can find the optimal values of these $x$ we can find the optimal values of

As an example suppose that $W_{e}=150$ ton, $a=2.4, H_{\Theta}=0.8 \mathrm{Md}$ (mega-dollars), $\mathrm{H}_{\mathrm{f}}=$ $0.8+0.03\left(W_{f}-W_{e}\right), \delta=0.08 \mathrm{yr}^{-1}, c=0.3+$ $0.1 \mathrm{x} g / w_{e}+0.9 x, \lambda=10^{-4} z^{-2.5}$ in $y^{-1}, B_{e}=1$, $\beta_{f}=\left(w_{f} / w_{e}\right)^{-1 / 2}, G(w)=0.02\left(w / w_{e}\right)^{2}$, and that in $x_{g} / x_{g}$ has the same distribution as in $\phi_{x, y, z}$ : normally distributed with expectation and standard deviation equal to 0 and 0.2 respectively. (In these data loads are in metric tons and money in megadollars.)

Using trial and error with the approach we have described we find the optimal values of $x g$ and $x$ in figs $4 a$ and $4 b$ respectively, for a given value of $p$.

These results can be interpreted in a number of ways. We may, for example, speak of an "equivalent" live load for earthquake resistant design. If we use as frame of reference an apparently consistent criterion of design, in which, the base shear would be proportional to $W_{f} 1 / 2$, we find the equivalent values of Wf displayed in fig 5 in terms of the actual Wf - We for the given value of $p_{\text {. This }}$ criterion would hold if the optimal rate of fallure were independent of $W_{f}$.

We could have solved the same problem for a different assumed form of the function $\beta_{f}$ and for different coefficients of $x_{5} / W_{e}$ and of $x$ in the expression that furnishes $C$, thus flnding different sets of equivalent live loads for a given $W_{f}-W_{\theta}$. Indeed, if we were considering the design of the auditorium's roof we would also find equivalent live loads on the roof, which would depend on the actual live load acting on the auditorium floor. This annoying situation raises the question of whether it is profitable to pursue the approach of computing equivalent loads which, when used in conjunction with a more conventional method of design, produce optimal designs. The answer is in the affirmative as a temporary measure, since acceptance of the present approach would be facilitated insofar as we shroud it with the appearance of conventional design formats. In the long rung however, we wil only be able to escape inconsistencies and unnecessary adjustments if we adopt an explicit optimization format. This we should attempt at a not much later stage in the evolution of building codes.

The foregoing analysis can be extended to structures that may undergo several states of damage and fallure.

\section{Two Stochastic Live Load Models}

A simpla way of incorporating the stochastic character of 1 ive load into the auditorium model is lo waive the assumption that ereaches its maximum shortly after completion of the structure. Since we are particularly interested in large values of $\alpha$ it is reasonable to assume that $\alpha \geq 1$, with $\alpha=1$ most of the time, and that this coefficient takes values greater than one for infinitely short time intervals according to a generalized Poisson process. For the sake of illustration we shall take the rale with which a given value of $\alpha$ is exceeded as

$$
\nu_{f g}=\nu_{0}^{e}-\frac{\alpha-1}{\alpha 0}
$$

where $\nu_{O}$ and $\alpha_{O}$ are constants. (In practice the parameters of $Y_{f g}$ depend on the size, mass, stiffnesses, and damping of the floor system under consideration.) We have chosen the symbol $\nu_{f g}$ for the rate of exceedance of $\propto$ because, If we write $\left.\left(x_{g}-w_{e}\right) \wedge W_{f}-w_{e}\right)$ for $\alpha$, eq 23 gives us the rate of structural failure due to gravity loads when the auditorium is ful1.

Under the present assumptions the probability of fallure at $t=0$ differs from $z e r o$ only with the auditorium empty. Hence, we must replace eq 19 with

$$
D_{\theta g}=\frac{\left(\mathrm{C}+\mathrm{H}_{\mathrm{e}}\right) \mathrm{P}_{\mathrm{e}}}{1-\mathrm{P}_{\mathrm{e}}}
$$

where subscripts eg refer to failure due to gravity loads when the auditorium is empty.

The total rate of failure is now $\nu_{\mathrm{fg}}+$ $\nu_{e s}+\nu_{f s}$, where the rates of failure due to earthquake when the auditorium is empty, $\nu$, or full $\gamma_{f s}$ are the same as in the deterministic live load model. Therefore, the expected loss due to failure is

$$
\mathrm{D}=\mathrm{D}_{\mathrm{eg}}+
$$

$\frac{H_{e} E_{e}+H_{f}\left[E_{f}+E\left(\nu_{f g}\right)\right]+C\left[E_{e}+E_{f}+E\left(\nu_{f g}\right)\right]}{1-\left[E_{e}+E_{f}+E\left(\nu_{f g}\right)\right]}$

The optimization process can now be pursued in the usual way.

As a second stochastic model for live load consider a situation that approximates those found on office and apartment building floors. We assume that, at every instant, the 1 ive load acting on any area is independent of that on every other area of the floors in question (see ref 17). We also assume that all the loads remain fixed over time intervals having an exponential probability distribution; in other words, in our model all live loads change simultaneously and do so according to a simple Poisson process; hence the load acting on a given section of the floors being considered may vary as shown in Fig. 6 .

The first of these assumptions has the following implications; a) at any given instant the expected load on an area $A$ is proportional to $A$ : b) the variance of this 
load is also proportional to $\mathrm{A}$; and $\mathrm{c}$ ) the probability distribution of this load asymptotically tends to be Gaussian as A tends to infinity, for positive values of the load.

Let $Q$ denote the probability that at any given instant the gravity load exceeds the structural capacity, and let $\mu$ denote the rate at which live load changes. Then the rate of structural failure under the sole action of gravity loads is $M Q$. If the consequences of failure did not depend on the magnitude of live load acting at the time of faijure, we could treat design optimization in the same way in which we dealt with the action of earthquakes on structures subjected to constant gravity loads, merely replacing $\nu$ with $\mu Q$.

Since the consequences of failure depend now on live load the expected present value of losses due to a single structural failure is

$$
D=E\left(\frac{\mathrm{H}_{\mu} \mathrm{Q}^{\mathrm{N}}}{\mu \mathrm{Q}+\delta}\right)
$$

instead of the value given by eq 11. Here $H$ is the loss incurred if failure takes place when the total load, W, is equal to the structural capacity to resist gravity forces, $x_{g} ; Q$ is the probability that $W$ exceeds $x_{g}$ at any given instant; and the expectation is taken with $\mathrm{X}_{\mathrm{g}}$ as random variable. If the structure is systematically rebuilt, the expected present value of the loss due to the ith failure is

$$
\left[E\left(\frac{\mu Q}{\mu Q+\delta}\right)\right]^{i-1} \cdot E\left[\frac{(C+H) \mu Q}{\mu Q+\delta}\right]
$$

including the cost of reconstruction, and the expected present value of the loss due to the entire sequences of failures is

$$
D=\frac{E\left[\frac{\left.(C+H)_{\mu Q}\right]}{\mu Q-\delta}\right.}{1-E\left(\frac{\mu Q}{\mu Q+\delta}\right)}
$$

In this treatment we have assumed that load effects are independent of the load distribution on the loaded area. When this is not the case we must replace the total load with its effect (generalized force) on the critical section or structural member. In structures that behave linearly the load effect is the integral, over the loaded area, of the load per unit area times the ordinate of the corresponding influence surface. If the probabilityl distribution of live load is independent of location, the expected liveload effect equals the expected unit live load times the mean influence ordinate, and times the potentially loaded area, while the variance of the live-load effect equals the variance of the unit-area live load times the mean squared influence ordinate and times the potentially loaded area. Owing to the relatively high probability there is that any portion of a floor be unloaded over a finite period of time, these means are to be computed over the area in which all the influence ordinates are positive and, separately, over that in which they are negative, and the two effects.positive and negative -- should be treated as if they affected different critical sections of structural members. The probability distributions of these effects are again asymptotically Gaussian as the corresponding potentially loaded areas tend to infinity.

Plastic structures can be treated in much the same way. Displacements associated with collapse mechanisms play the role of influence ordinates while the work done by yield moments at plastic hinges plays the role of the generalized forces in structures of linear behaviour. (See ref 18 for an application to the static limit design of slabs under random live load.)

The dependence of the parameters of the probability distribution of live load on location relative to walls and partitions is probably not negligible for design purposes (19) This dependence can also be incorporated in the model.

This second stochastic model of live load can be combined with the generalized Poisson process that idealizes earthquake effects, in much the same way as we combined this process with the first stochastic model.

A more realistic idealization would treat live load as the superposition of two interdependent stochastic processes. One process would correspond to furniture loads and the other to loads due to people.

\section{Closing Remarks}

If earthquake engineering is to be a rational discipline it must aim at the making of optimal decisions. In many instances, as when drafting a building code, the engineer's utility scale coincides. with that of society, and as a professional he can never drift greatly away from society's scale of utilities. Hence, even in cases where there may be conflict of objectives it is reasonable to begin by formulating optimization criteria from the viewpoint of society.

Implicit and indirect optimization in the guise that has led to present building code provisions and established practice is unsatis. factory because it requires a number of adjust. ments, sometimes contradictory, and because, by obscuring the true issues, it tends to produce designs that lie far from the optimum solutions. However, several steps must be taken before explicit reliability optimization can be implemented $(12)$. One series of steps concerns the education of the engineering profession to make this approach palatable. Another concerns the production and diffusion of design aids, including computer programs and reliability-optimization computer languages. Since the present methods for sensing and interpreting the wishes of society are defect. ive, explicit optimization will doubtless also require that interdisciplinary groups participate in the process, as the engineering profession should not attempt to hold a monopoly on this function.

Apparently, before explicit reliability optimization is adopted we should also gather vast statistical information on earthquake characteristics and other disturbances as well as on structural properties and on the consequences of failure. Scarcity of this information is not, however, a valid reason for postponing the adoption of the type of code format and of design practice proposed, since this approach merely makes that scarcity more 
obvious, while it is just as real even if less noticeable in the present versions of codes and established practice. The same applies to the development of methods of analysis and design leading to reliability optimization. And the sooner we attempt this approach the earlier in the game we will identify the areas in which we are most ignorant and in which a research effort would be most profitable.

The present paper develops some simplified methods of reliability optimization applicable to earthquake resistant design. Its main emphasis is in the combination of effects of gravity loads and seismic forces. It is hoped that it will stimulate research along these same lines and in the gathering of statistical data which will permit improving the stochastic models adopted.

\section{Acknowledgement}

I am grateful to Dantón Gutiérrez-Lemini, Armando Flores-Victoria, Enrique Mendoza, and Alonso Bretón for their valuable contributions to this paper and to Daniel Ruiz and Luis Esteva for their critical review of the manuscript.

\section{Refarences}

1. Vanmarcke, E. H, Hirst Passage and other Failure Criteria in Narrow Band Random Vibration: A Discrete State Approach," Research Report R69-68, Dept. of Civil Engrg,, Mass. Inst. of Techn. (1969).

2. Barlow, R. E. and Proshan, F., Mathematical Theory of Reliability, John Wiley and Sons, Inc., New York, 1965.

3. Esteva, L., "Seismicity Prediction: A Bayesian Approach," Proc. Fourth World Conf. on Earthq. Engrg., Santiago de Chile (1969): pp A1: 173-84.

4. Rosenblueth, E. and Bustamante, J. I., "Distribution of Structural Response to Earthquakes," Proc. Am. Soc. of Civ. Engrs., 88, EM3 (June 1962), 75-106。

5. Newmark, No M. and Rosenblueth, E. Fundamentals of Earthquake Engineering, to be published by Prentice-Hall, Inc., Englewood Cliffs, N.J., 1971.

6. Esteva, L., "Consideraciones Prácticas en la Estimación de Riesgo Sísmico," Instituto de Ingeniería, 248 (Apr. 1970).

7. Wood, L. W., "Factor of Safety in Design of Timber Structures," Trans. Am. Soc. of Civ. Engrs., $125(1960), 1033-45$.

8. Allen, D. E., Probabilistic study of Reinforced Concrete in Bending, Natl. Res. Council of Canada, Division of Building Research, Techn. Paper311 (January 1970).

9. Rosenblueth, E. and Mendoza, E. "Reliability Optimization in Isostatic structures," submitted for publication in the Journal of Engineering Mechanics Division, Am.Soc. of Civ. Engrs., 1971

10. Moses, F., "Approaches to structural Reliability and optimization," An Introduction to structural optimization, Solid Mechs. Division, Univ. of Waterloo, Waterloo, Ontario, Canada, Study 1, 1969, pp 81-120.

11. Rosenblueth, E., Esteva, L。, and Damy, J.E.,
"Bonus and Penalty to Price of Concrete", submitted for publication in the Journal of the Construction Division, Am。 Soc. of Civ。 Engrs., 1971.

12. Rosenblueth, E., and Esteva, L., "Reliability Basis for Some Mexican Codes," presented at the Am. Concrete Inst. Symposium on Probabilistic Methods, Denver, March 1971.

13. Esteva, Lo and Rosenblueth, E., "Use of Reliability Theory in Building Codes", to be presented at the Conference on Applications of Statistics and Probability to Soil and structural Engineering, University of Hong Kong, September 1971.

14. Blume, J. A., "structural Dynamics in Earthquake Resistant Design," Trans. Am. Soc. of Cive Engrs., $125(1960)$, 1088-1139.

15. Benjamin, J. R. and Williams, Ho A., "The Behaviour of One-Story Brick Shear Walls," Proc. Am. Soc. of Civ. Engrs., 84, ST 4 (July 1958), 1723-30.

16. Esteva, L., Behaviour under Alternating Loads of Masonry Diaphragms Framed by Reinforced Concrete Members", Symposium on the Effects of Repeated Loading on Materials and Structural Elements, RILEM, Mexico, 1966 .

17. Rosenblueth, E., "Teoría de la Carga Viva en Edificios," Ingenierí, Mexico, 29, 4 (Oct. 1956), $51-72$.

18. Arean, F., "Estudio de la Carga Viva en Edificios," Professional thesis, Esc. Nac. de Ing., Univ. Nac. Aut, de Mexico, Mexico, 1956.

19. Lind, N. C., and Davenport, A. G., "Towards Practical Application of structural Reliability Theory, "Solid Mechanics Division, Univ. of Waterloo, Canada, 73 (Feb. 1971)。 


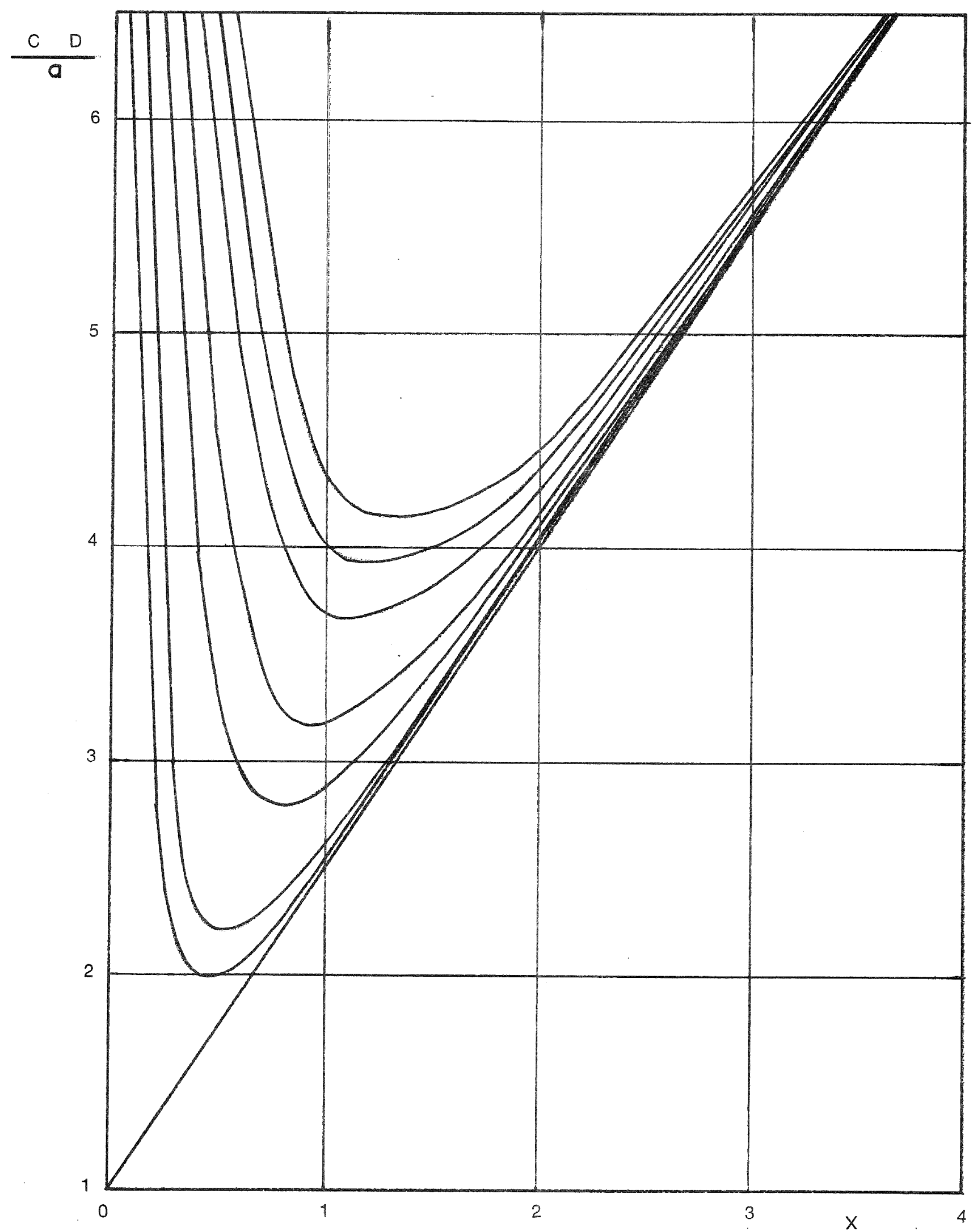

FIG 1. OBJECTIVE FUNCTION WITH CONSTANT GRAVITATIONAL LOADS 


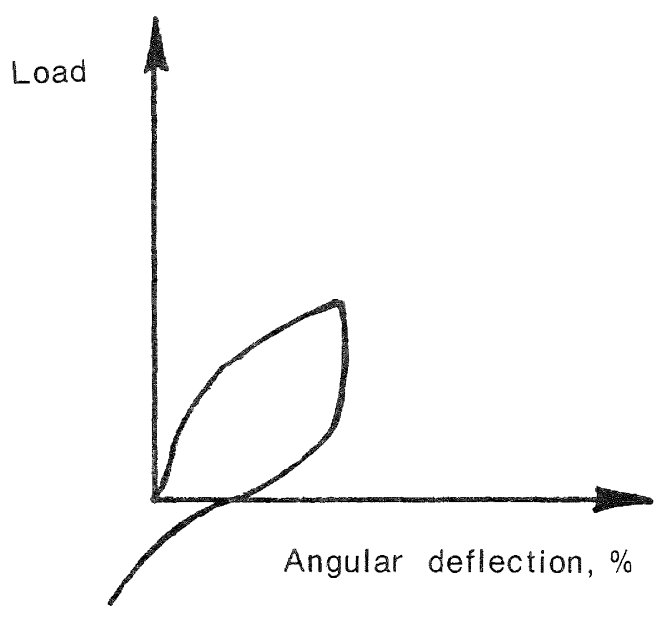

FIG. 2a. FORCE DEFORMATION CURVES FOR ENFRAMED SHEAR WALLS

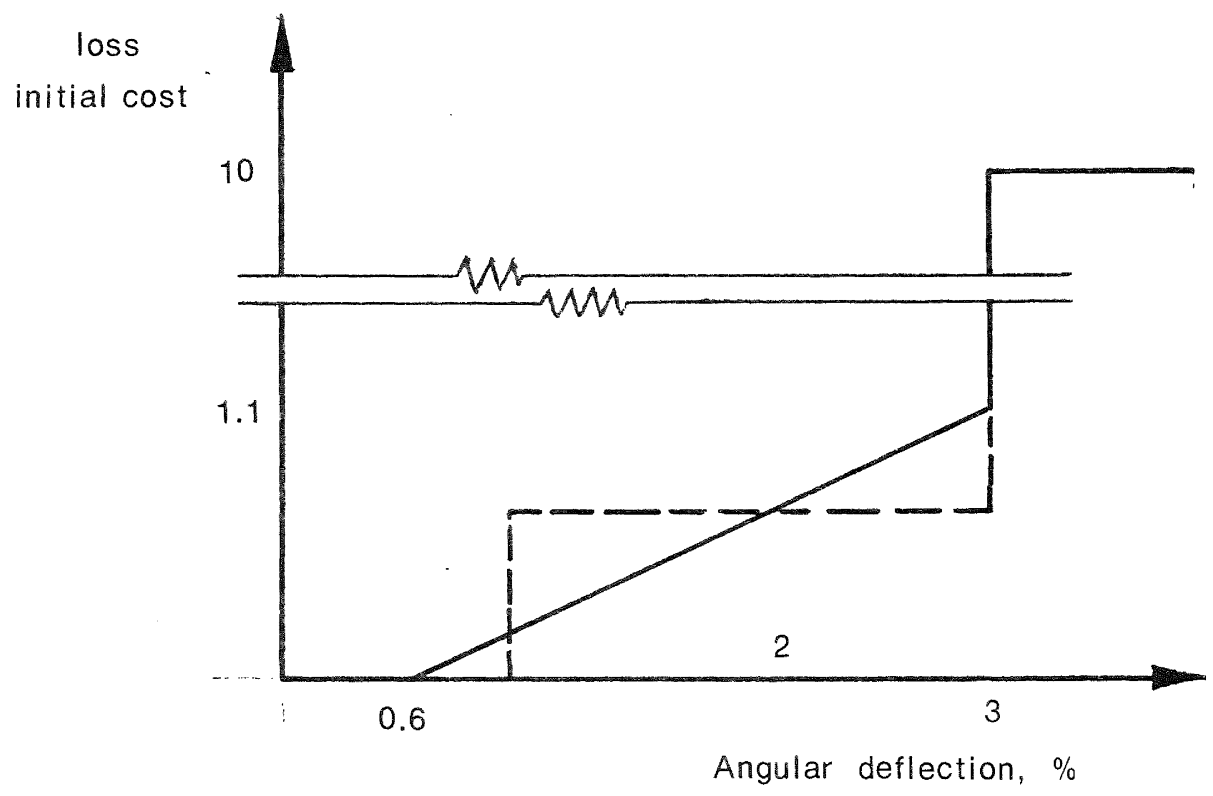

FIG 2b. LOSSES DUE TO DAMAGE AND FAILURE OF SHEAR WALLS

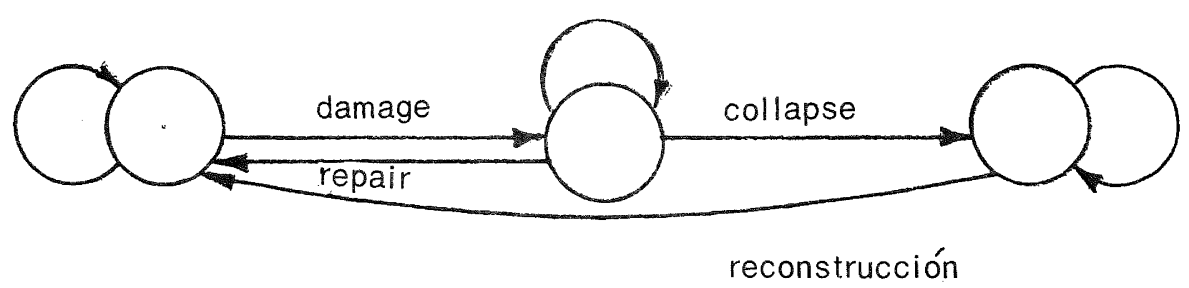

FIG 3. STRUCTURAL STATES AND TRANSITIONS 


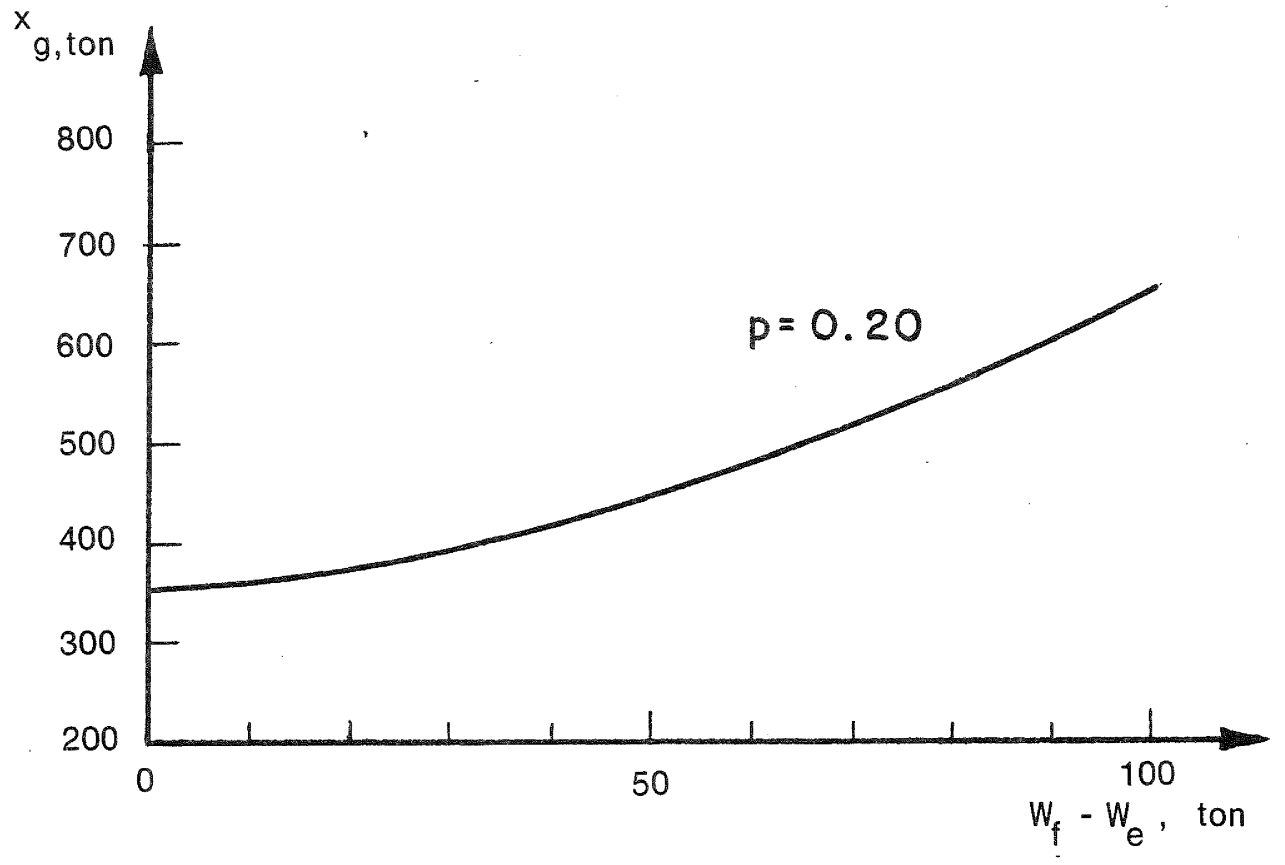

a)

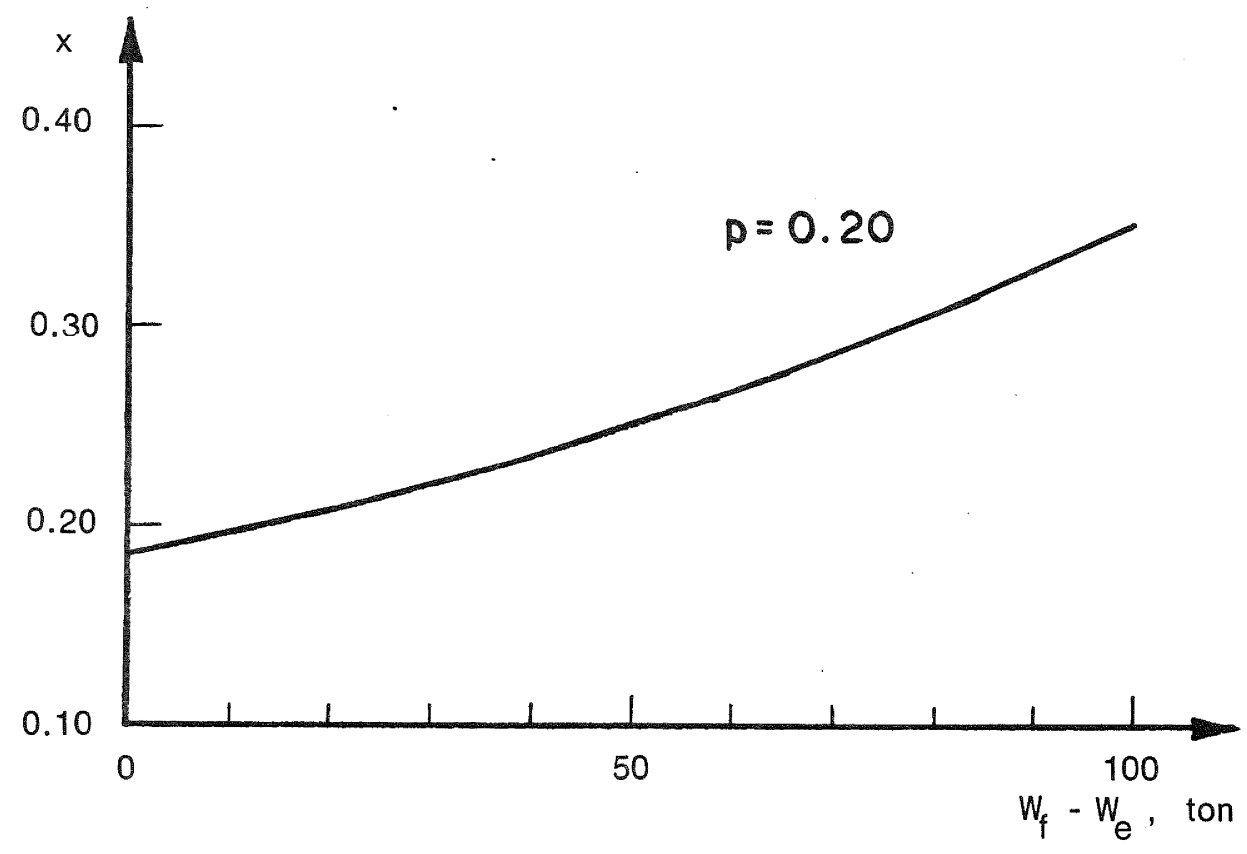

b)

FIG 4. OPTIMAL VALUES OF DESIGN PARAMETERS IN AUDITORIUM - TYPE STRUCTURE 


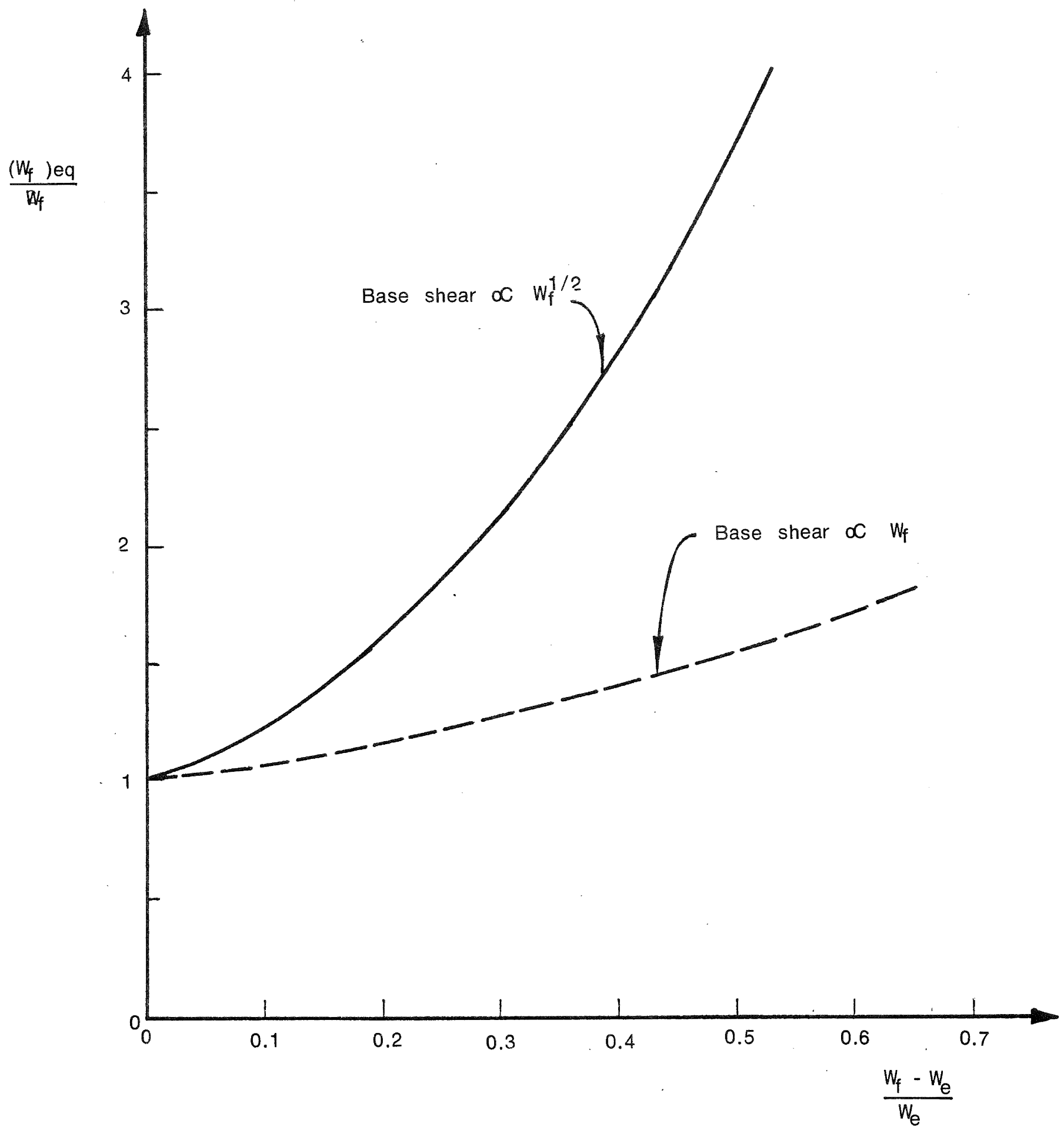

FIG 5. EQUIVALENT DESIGN LIVE LOADS 


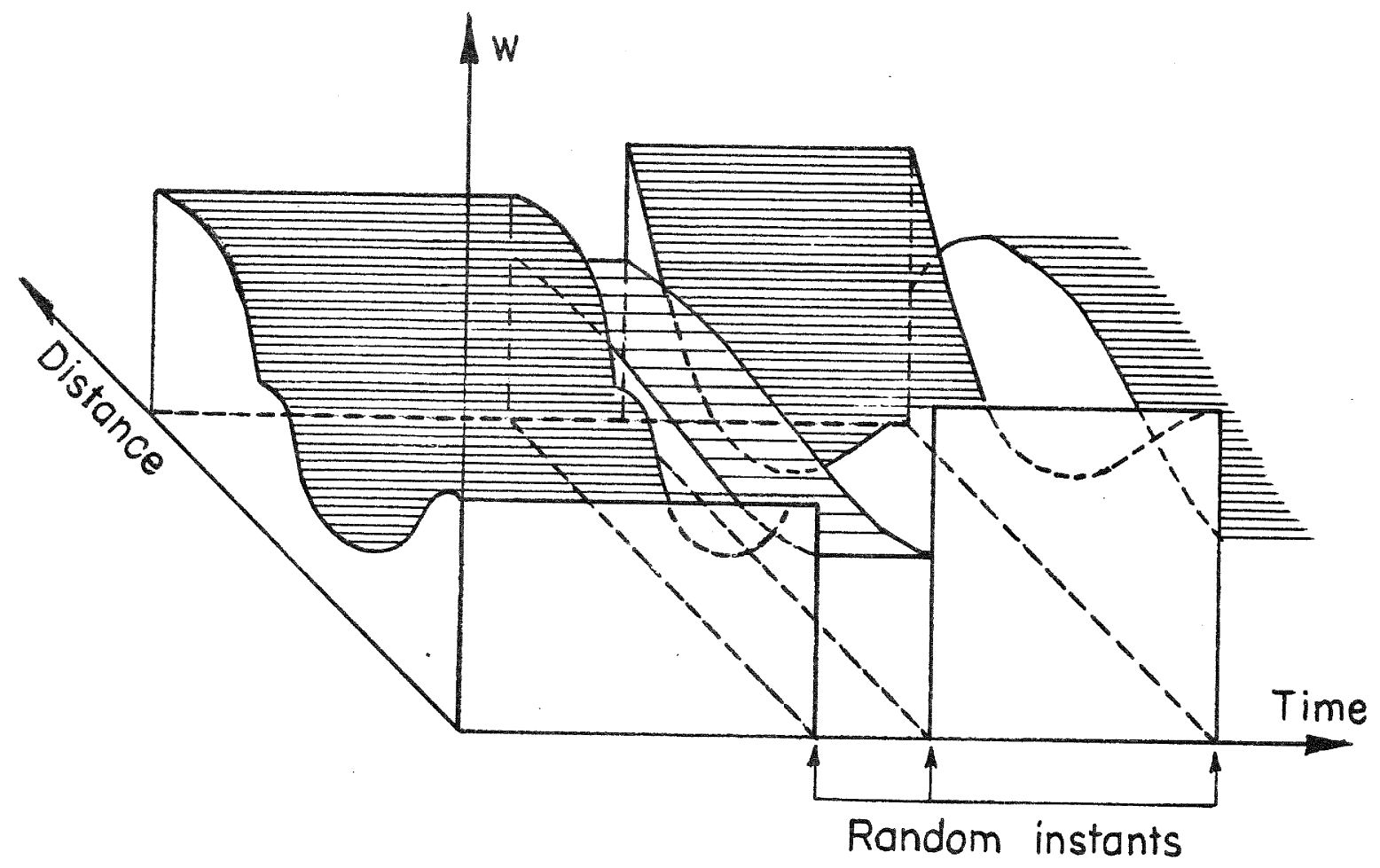

FIG 6. LIVE LOAD DISTRIBUTION ALONG A FLOOR CROSS SECTION. 